\title{
A Modified Gravitational Explanation of the Dynamic Origin of the Stars' Rotation and Revolution
}

\author{
Wei Fan (凡伟) \\ Yunnan Institute of Modern Physics Kunming, 65000 China
}

\begin{abstract}
:
Because Newton's gravity and Einstein's general theory of relativity are macroscopic gravitational theories, therefore, this paper attempts to establish a set of quantum gravity theory of the microscopic expression of Newton's and Einstein's theory of gravity to make up for the shortcomings of the existing macro-gravity theory at the micro level, and further develop the macroscopic gravity theory into the microscopic field. Based on the cognition of the field theory model, from the general assumption, space-time is further regarded as an ideal fluid, from the perspective of the distribution of ideal fluid density, this paper derives the Newton's universal gravitational equation and Einstein's general relativity equation. On the micro level, the gravitational field is further interpreted as a gradient field of space-time density; the Newtonian potential is further interpreted as the density of an object at the micro level; gravity is further interpreted as the potential pressure(space-time pressure) exhibited by the gradient of the density of the object at the micro level; flection space-time is further interpreted as the embodiment of the gradient distribution of the ideal fluid (space-time) density at the micro level. Based on a modified gravitational model, it explains the dynamics of the stars' rotation and revolution.
\end{abstract}

Keywords: gravity; dark matter; dark energy; Newtonian mechanics; motion

\section{1: Introduction}

What is the origin of dark matter? What is the origin of dark energy? What is the origin of matter? What is the origin of space? What is the origin of gravity? What is the origin of the motion? What is the origin of inertia? What is the origin of the orbital elliptical orbital motion? What is the origin of the dynamics of the rotation and revolution of the stars? What is the origin of the free fall movement? This is some unsolved mystery of contemporary physics. So, this article tries to solve these problems. At the same time, by consulting the relevant literature, many people try to explain these similar problems in various ways $[1,2,3,4,5,6,7,8,9,10,11,12,23]$. However, this paper only considers the interpretation of these problems from the perspective of Newtonian mechanics and general relativity.

Since Newton's theory of gravity and Einstein's general theory of relativity are both macroscopic theory of gravity, this paper attempts to seek a microscopic expression theory of macroscopic gravity theory to obtain a more basic interpretation of the origin of gravity.
At the same time, there are currently two sets of theories describing motion, one is Newton's vector mechanics theory that describes motion from a force perspective; the other is an analytical mechanics theory that describes motion from the perspective of energy. Although both sets of theories describe motion, they cannot interpretation the origin of the movement. Also because the origin of motion cannot be interpreted, this leads us to not interpretation the origin of inertia, ellipsoidal orbital motion, star rotation and revolutionary dynamics through Newtonian mechanics and analytical mechanics. These are some common pain points in these two sets of theories.

Among them, because Newtonian mechanics describes motion in terms of force, and the discussion of force must have a force exertor, which cannot avoid the existence of God, this is another pain point of Newtonian mechanics. Therefore, from the perspective of real history, Newtonian mechanics is a theological theory that sums up the universe to satisfy God's design and God's first impetus by summarizing natural laws and phenomena. It is not a 
set of scientific theories in the true sense, and Newton is not a scientist, but a theologian. This important reason is because Newton's era is the era of creationism. In that era, there was only theology and no science; After Newton was born two centuries, Darwin subverted the creationism, so Newton himself is certainly not willing to accept the strange name of scientists; In addition, Newton did not study theology in his later years, and Newton had studied theology throughout his life. That is to say, Newtonian mechanics is a part of theology, and Newtonian mechanics is called scientific theory, just because we deliberately avoid the theological thoughts expressed by Newton and only discuss the natural laws of satisfaction. Of course, although Newtonian mechanics is part of theology, we cannot deny his contribution to the study of natural laws for modern science.

In addition, although analytical mechanics is also called mechanics, analytical mechanics describes motion in terms of energy, and does not use force, so it jumps out of the mechanics framework and can effectively avoid the existence of God's first driving force. This is a big step forward in analytical mechanics. But considering that analytical mechanics is a mathematical analysis theory, energy is just a mathematical abstraction expression, not a physical object. Therefore, analytical mechanics lacks corresponding physical interpretation, so analytical mechanics is very abstract and difficult to understand. This is another pain point of analytical mechanics.

Based on such historical background, this paper attempts to improve their conceptual foundations by introducing field theory based on the physics principles of Newtonian mechanics and analytical mechanics, so as to describe and interpretation the origin of motion, and then interpretation origin of inertia, ellipsoidal orbital motion, star rotation and revolutionary dynamics, thus overcoming these pain points in Newtonian mechanics and analytical mechanics, and finally established a new paradigm theory independent of Newtonian mechanics and analytical mechanics.

\section{2: A new physics model will be built}

Throughout the history of science, there was no one has ever tried to explain the origin of matter, space and gravity, and there was no one has ever tried to unify them (even most people think this is a philosophical question that never has an answer, but this is not true). Here, I will try to construct a unified model of material, space and gravity and explain their origins.

Based on the general theory of relativity [3], gravity is a manifestation of space-time bending, so the essence of space can be regarded as the gravitational field. At the same time, considering the size of the Newtonian potential is a quantity, and the amount of the quantity can be expressed by the density distribution, so we can use the density to express the Newtonian potential. Therefore, we can think of the gravitational field as a gradient field of the density of an ideal fluid $\Delta \varphi=f$. Such, for material, space and gravity models, we can construct models for matter, space and gravity as following (assumptions and definitions): there exists a minimum unit for all matters, which we defined as : ground substance, labeled as $d v$; the space $S$ is made up of ground substance $d v$, which is: $S=\iiint_{\Omega} d v$, this means that the ground substance is the smallest unit of matter common to matter and space, the space condenses form matter, so $M=S \cdot k=\iiint_{\Omega} d v \cdot k$, where $k$ is the coefficient of condensation, so we can that the space is the ground-substance form of matter, $S=\iiint_{\Omega} d v=\frac{M}{k}$,

and matter is the condensed form of the space $M=S \cdot k$; as there is a universal density of the ground substance for the universe which we defined as 1, and as matter is formed by the ground substance, $M=\iiint_{\Omega} d v \cdot k$, which leads to a decrease in the density of the ground substance around the 
matter formed, $\rho \prec 1$, and this lead to a linear change in the density of the ground substance, $\nabla \rho \propto \nabla u$, the gradient of change in the density equals the gradient of the change in gravitational potential, $\nabla \rho=\nabla P$, thus, the origin of gravity is gradient of change in the density of the ground substance which forms matter, for short: space - gradient, so the gravitational field is a field of the space potential. In this way, the field strength $E$ is proportional to the divergence $\nabla \cdot \vec{A}$ of the density $\rho$ of ground substance $d v$, which can be written as:

$$
E \propto \operatorname{div} \vec{A}=\nabla \cdot \vec{A}
$$

Thus, the gravitational field is not radial but contracted. So the gravitational field is a negative-source field, $\operatorname{div} \vec{A}<0$. Negative-source means the field comes from the high altitude instead of the centre of the earth.

Thus, the gradient of gravitational potential equals gradient of the density of ground substance of free space.

$$
\nabla P=\nabla \rho
$$

From the above formula, I use density to explain the origin of the potential, and use the gradient of the density of the matrix to explain the origin of gravity (flection time-space), so we propose a feasible interpretation of the origin of gravity. Also, because gravity is a negative source field, gravity is not from the tension of the object, but from the potential pressure (space-time repulsion or space-time pressure) at high altitude. And this dark energy can be explained by the space-time repulsion, so dark matter can be interpreted as space-time, and dark matter density is space-time density. Thus, the origin of the expansion of the universe is due to space-time repulsion.

As free space is made up of ground substance, and the ground substance forms a linear distribution, thus, we can also say, the gradient of free space equals the gradient of gravitational potential, which is expressed as:

$$
\nabla P=\nabla \rho=\nabla V
$$

From the equation above, the source of space is still a field of gravitational potential, so space is not flat, but shows a linear distribution, meanwhile, the macro universe is the result of combining an infinite number of gravitational fields.

Thus, the strength of the gradient of gravitational potential equals the strength of gradient of space , considering the strength of gradient of gravitational potential is the negative gradient of gravitational potential, $E=-\nabla P$. Therefore, the strength of space gradient field is the negative of the gradient of gravitational potential.

$$
E=-\nabla V=\nabla \rho=-\nabla P
$$

Due the fact that the gravitational potential is indeed space potential. (In the following expressions I will use space potential to substitute gravitational potential).

Therefore, from Newton's mechanics, the relationship between the magnitude of space potential and mass and distance can be expressed as:

$$
P=-G \frac{M}{r}
$$

Substitute ( 5 ) into ( 4 ), we can deduce the relationship between the strength of space gradient field and mass and distance:

$$
E=-\nabla V=-\nabla \rho=-\nabla P
$$

From this, the strength is proportional to the mass of the body, and is inversely proportional to distance. As gravitational potential is space potential, then, for the potential energy for a test mass in the space can be expressed as:

$$
E_{P}=m g h
$$

As gravitational potential energy is spatial potential energy (P.S: We will use spatial potential energy to 
substitute gravitational potential energy in the following passage), Then the relationship between spatial potential energy and distance can be expressed as :

$$
E=-\frac{G M m}{r}
$$

The interaction of spatial gradient potential between two bodies fulfills the Newton's law of universal gravity.

$$
\vec{F}=-G \frac{M m}{r^{3}} \vec{r}
$$

It can be seen from the above formula that the origin of gravitation is the universal potential pressure or the universal space potential gradient, we use gradient of spatial potential or the gradient of spatial density) to explain gravity. The interaction between two bodies is the interaction between the gradient of spatial potential of two bodies, and the magnitude of the interaction is proportional to the mass of the body, and inversely proportional to the square of distance. Therefore, I derived Newton's law of gravity from the perspective of time and space pressure. So, you can see that gravity is not a basic force, but a macroscopic phenomenon expressed by positional pressure. Which is saying that free fall of an apple is due to the thrust instead of the attraction from the earth. This can solve the question that why gravity in Newton's theory can act on an infinite distance.

It can be seen from the above derivation that space-time is interpreted as an ideal fluid, and the curved structure of space-time depends on the distribution of space-time density, so the pressure $T^{\mu v}$ generated by the distribution of space-time density can be expressed as:

$$
T^{\mu v}=(\rho+p) u^{\mu} u^{v}+p g^{\mu v}
$$

Where $\rho$ is the space-time density, $p$ is the pressure,

and $u^{\mu}$ is the four-dimensional velocity. At the same time, it can be seen that the space-time pressure $T^{\mu \nu}$ here is the energy-momentum tensor $T^{\mu \nu}$ of the GR. When using Riemannian geometry to express this space-time pressure, we can get the general relativity equation:

$$
R_{\mu \nu}-\frac{1}{2} g_{\mu \nu} R=\frac{8 \pi G}{c^{4}} T_{\mu \nu}
$$

It can be seen from the above that space-time bending is the embodiment of the space-time density distribution. That is to say, general relativity can be regarded as a local (geometric) approximation of a more basic gravitational theory, or general relativity is a geometric expression (interpretation) of a more basic gravitational theory.

Conclusion: Through the above proof, I found that the space is composed of the matrix (also the gravitational field), the matter is the condensed state of space, and the space is the matrix state or the diffused state of matter. Gravity is not a basic force, but a macroscopic potential pressure phenomenon represented by the gradient of spatial density, this gives a microscopic interpretation of the origin of gravity. At the same time, we also use space-time to explain the origin of dark matter, and use the space-time repulsion to explain the origin of dark energy. This can explain the origin of Newtonian gravity, Einstein's general relativistic flection space-time and Erik Verlinde's entropy force [21,22,23]. Among them, Newtonian gravitation is the algebraic expression of the potential pressure(space-time pressure) generated by the gradient of spatial density; general relativity is the expression of the Riemannian geometry of the potential pressure generated by the gradient of spatial density; the Erik Verlinde's entropy force is a potential pressure(space-time pressure) phenomenon represented by the gradient of spatial density.

\section{3: According to the new physics model, some questions will be thoroughly explained.}

\section{1: The origin of motion, velocity, acceleration}

We all know that a body can perform motion. But why is that happening?? What causes the motion? We cannot give an answer. Classical 
physics only gave a mathematical description , but had never tried to give a physical explanation or definition. In general, when the position of an object changes, we say this object can move.

In classical mechanics, position vector for an object in a flat space is:

$$
\vec{r}=x \vec{i}+y \vec{j}+z \vec{k}
$$

According to the general relativistic micromodel, as the space is not flat, but forms a gradient distribution, then, the position vector should be changed to a vector under the gradient space, which is equivalent to calculate the gradient of the density of the position vector in the flat space:

$$
\nabla r=\nabla \rho=\left(\frac{\partial}{\partial x} \vec{i}+\frac{\partial}{\partial y} \vec{j}+\frac{\partial}{\partial z} \vec{k}\right) \rho
$$

$\nabla$ indicates the gradient space, $\nabla r$ which represents the bit vector under the gradient space, will be the same below.

Considering the kinematic equation in the flat space:

$$
\vec{r}(t)=x(t) \vec{i}+y(t) \vec{j}+z(t) \vec{k}
$$

As the space is not flat, but forms a gradient distribution, Thus, the real kinematic equation should describe the motion of an object in the gradient space. Therefore, the motion of an object in the gradient space can be described as:

$$
\nabla r(t)=\nabla \rho(t)=\frac{\partial \rho}{\partial x}(t) \vec{i}+\frac{\partial \rho}{\partial y}(t) \vec{j}+\frac{\partial \rho}{\partial z}(t) \vec{k}
$$

Equation (14), in classical mechanics, is a function of coordinates about time, which is only a mathematical expression without any actual physical meaning; From equation (15), in the gradient space, the motion of the object represents the change of the potential of the object with time. This means that the origin of motion is the expression of the change of the potential of the object, thus explaining the origin of the motion.

Since motion is a manifestation of a change in the potential of an object, the motion of the object requires the potential pressure $\nabla U$ to be pushed, so that the motion of the object requires the potential difference $\nabla U_{A B}=\nabla \varphi_{A}-\nabla \varphi_{B}$ to be maintained, so that the object does not move on the equipotential surface. The mathematical expression is:

$$
\left\{\begin{array}{l}
\sum_{i} \nabla U_{A B}=\nabla \varphi_{A}-\nabla \varphi_{B}=0 \Rightarrow \frac{d \nabla \rho}{d t}=0 \\
\sum_{i} \nabla U_{A B}=\nabla \varphi_{A}-\nabla \varphi_{B} \neq 0 \Rightarrow \frac{d \nabla \rho}{d t} \neq 0
\end{array}\right.
$$

In this way, we have come up with a definition of the origin of the motion. This can be used to explain the origin of the orbital elliptical orbital motion. Because the object does not move on the equipotential surface, when the star is subjected to the force of other stars, its orbital potential changes and appears as an elliptical orbital motion. Considering that inertial motion is a kind of motion, because motion requires positional pressure to push and maintain, so, motion is not an inherent property of an object. Even if the object is in an ideal state without any force, the object will not move linearly at a constant speed.

Generally, in classical theory, we do not know the origin of velocity, but only the mathematical expression which has no physical meaning. That is expressed as:

$$
\vec{v}=\frac{d \vec{r}}{d t}
$$

Deduced from (13), the position vector under gradient space $\nabla r=\nabla \rho$, then velocity has its own physical meaning in the gradient space, which is:

$$
\vec{v}=\frac{d \nabla \rho}{d t}
$$

It can be seen from the above that the magnitude of the object velocity in Newtonian mechanics represents the rate of change of space potential or density over time in the gradient space, so that we have physically defined the velocity for the first time, explaining the origin of velocity. From the theory above, only when the spatial potential of an object changes then the object will have velocity and perform motion. 
In general, in classical physics, we do not know the origin of acceleration, and our definition do not has any physical meaning, which is:

$$
\vec{a}=\frac{d \vec{v}}{d t}
$$

In the gradient space, as $\vec{v}=\frac{d \nabla \rho}{d t}$, therefore, the acceleration of a body can be expressed as:

$$
\vec{a}=\frac{d^{2} \nabla \rho}{d t^{2}}
$$

From the equation above, the acceleration of a body expresses the rate of change in the rate of change of the spatial potential or spatial density. Thus, we defined acceleration for the first time and explained the origin of it.

\section{2: The origin of the law of inertia}

From Newton's first law, we know that everything has inertia, which is: "An object does not need any external force to maintain its current state of motion. An object will stay still or move in a straight line with constant speed." However, what is the origin of inertia? Why no external force is required to keep the state of motion of an object? Why will an object keep moving in a straight line ? This has always been a mystery.

According to the proof above, motion is not an intrinsic property of an object. Therefore, for inertial motion, when the external force is 0 (or the object is not subjected to external force), the object will only remain relatively static and will not move linearly at a constant speed. The mathematical expression is:

$$
\sum_{i} \vec{F}_{i}=0 \Rightarrow \vec{v}=\frac{d \nabla \rho}{d t}=0
$$

Thus, we redefine the inertia. As can be seen from the above, the motion of the object requires force to maintain.

Considering that inertial motion is a kind of motion, we can give the equation of motion and velocity equation of inertial motion. The mathematical expression is:

$$
\left\{\begin{array}{l}
\nabla r(t)=\nabla \rho(t) \\
\vec{v}=\frac{d \nabla \rho}{d t}
\end{array}\right.
$$

According to the above, we know that motion is a phenomenon in which the spatial potential of an object changes, and the object does not move on the equipotential surface.

Considering that the motion of the object requires the potential pressure to push, in order to facilitate understanding, we can understand the potential pressure into kinetic energy, because changing the potential pressure of the object is to change the gravity potential energy of the object $E_{p}=m g h$, because the origin of the potential energy is kinetic energy, the kinetic energy is a kind of expression of the potential energy, can be mutual transformation, so the space potential energy is the kinetic energy at the same time here $E=\frac{1}{2} m v^{2}$. Considering that the energy required to maintain an object's inertial motion is the energy required to resist gravity, we can define this energy in a special way: dynamic potential energy. The mathematical expression is: $E=m g h=\frac{1}{2} m v^{2}$ Therefore, we can also draw the conclusion that "the motion of an object needs dynamic potential energy (kinetic energy) $E=m g h=\frac{1}{2} m v^{2}$ to maintain. When the kinetic energy of the object is 0 , the object always remains at rest and does not move linearly at a constant speed. The mathematical definition is:

$$
\left\{\begin{array}{l}
E=m g h=\frac{1}{2} m v^{2} \succ 0 \Rightarrow v \succ 0 \\
E=m g h=\frac{1}{2} m v^{2}=0 \Rightarrow v=0
\end{array}\right.
$$

Where, $E=m g h=\frac{1}{2} m v^{2}$ is the dynamic potential energy, $v$ is the velocity, so we give a new definition of inertial motion. It can be seen from the above formula that inertia is the buffering process of the kinetic energy of an object against the 
consumption of gravity. Thus, I explained the origin of inertial motion.

Discussion: Due to the limitations of the era of cognition. The era of Galileo and Newton could not explain the origin of the movement. Therefore, they did not know that movement is the embodiment of the change of the position of the object. It is not known that the movement needs a kind of potential pressure (kinetic energy) to maintain, which leads them to misunderstand that the movement is an intrinsic property of the object, so it leads to the incorrect definition of inertia. Also, because the Galileo era does not have the concept of gravity, nor the origin of friction, Galileo does not know that the moving object is subject to gravity, and also unknown that friction is a manifestation of the kinetic energy consumed by gravity on the contact surface. It is misunderstood that the object will continue to move without friction; also, because the Newton era has no concept of kinetic energy, Newton does not know that the motion of the object requires kinetic energy to maintain, and finally causes Newton to follow the wrong view of Galileo.

Through the above proof, due to the existence of gravity, any object will be consumed by gravity during the movement. Therefore, the inertial motion is the buffering process of the kinetic energy of the object against the gravity consumption. When the external force of the object is 0 (or the kinetic energy is 0) Time), the object will only remain relatively static and will not move linearly at a constant speed.

Summary: Through the above proof, we can find that motion is the embodiment of the change of the potential of the object. The motion of the object requires force or kinetic energy to maintain. The motion is not the original nature of the object, even if the object is in an ideal state without any force, the object will not move linearly at a constant speed. In addition, we all know that the law of inertia has never been verified by experiments, nor can it be verified by experiments. Physics is an experiment-based science. The theory that has not been experimentally verified can only be conjecture; the theory that has not been confirmed by experimental verification is not scientific theory. Therefore, Galileo and Newton's definition of inertia is fundamentally unfounded.

\section{3: Origin of force}

At the same time, there has never been a definite definition of the origin of force in the history of science, so we do not know the origin of force all the time.

According to Newton's second law $\vec{F}=m \vec{a}$, because the acceleration is the object of the spatial potential of the rate of change of rate of change over time in the gradient space $\vec{a}=\frac{d^{2} \nabla \rho}{d t^{2}}$, the force can be defined as: the quality of the object and the space of potential over time and the rate of change of the rate of change of the product, i.e., the space of the object to have potential gradient variation of the rate of change of size. Expressed as:

$$
\vec{F}=m \vec{a}=m \frac{d^{2} \nabla \rho}{d t^{2}}
$$

It can be seen from the above formula that force is a mathematical quantitative concept and does not exist as a special object, that is to say, force does not exist. It can also be seen that a force applied to an object is changes the space potential of the object. Therefore, force is an action potential energy.

When the rate of change of the space potential of an object maintains a constant rate, i.e. $\vec{a}=\frac{d^{2} \nabla \rho}{d t^{2}}=\vec{g}$. It can explain the free fall movement, and the force can be expressed as:

$$
\vec{F}=m \vec{a}=m \frac{d^{2} \nabla \rho}{d t^{2}}=m \vec{g}
$$

It can be seen from the above equation that the motion principle of free fall is the same as that of 
conventional motion.

Conclusion: according to the above derivation, we have given the exact physical definitions of motion, velocity, acceleration, inertia and force respectively from the perspective of the space potential, and explained their origin.

\section{4: Further derivation and new prophecy}

\section{1: origin of star (earth) rotation}

From the above derivation, it can be found that the essence of motion is that the space potential increases after the object is subjected to the action amount, generating a momentum of potential, which causes the space potential to change with time, expressed in a sporty way, mathematically expressed as: $\vec{v}=\frac{d \nabla \rho}{d t}$. So, to the rotation of the object, the object of irregular shape and density distribution is not uniform, causing the astral center and geometric center don't overlap, as the focus of stars around other stars of perturbation (action), can let a focus to create a velocity, displacement of center of gravity of the stars is expressed in the form of spin, mathematical expressions for the:

$$
\vec{v}=\frac{d(\nabla \rho \pm x)}{d t}
$$

Where, $v$ is the velocity, $\nabla P$ is the center of gravity potential, $t$ is the time, $x$ is the perturbation potential. It can be seen that when the star is slightly disturbed and $x$ changes, the center of gravity velocity of the star will change, and this change of center of gravity velocity will be reflected by the rotation of the star. The trajectory can be expressed by the equation of motion $\nabla r(t)=\nabla \rho(t)$.From this, we can see that the dynamic origin of the rotation of stars comes from the perturbation of other stars around them. We can also see that the rotation of stars does not require god's first impetus.

\section{2: origin of star revolution and elliptical orbit motion}

Because the origin of motion is the embodiment of the change of the potential of the object, for the orbital rotation of the star, when the orbital potential of the star is disturbed by other surrounding stars, the orbital potential of the star will change, and the disturbed change of the orbital potential of the star It will be expressed in the form of a revolution. The mathematical expression is:

$$
\vec{v}=\frac{d(\nabla \rho \pm x)}{d t}
$$

Where, $v$ is the velocity, $\nabla \rho$ is the orbital potential, $t$ is the time, $x$ is the perturbation potential. From this we can see that the perturbation of the orbital potential of a star is expressed in the form of revolution. Where, its motion trajectory can be expressed by the motion equation $\nabla r(t)=\nabla \rho(t)$, from which we can see that the dynamic origin of star revolution is from the perturbation of other nearby stars. So, we can see that the revolution of the stars does not require or have the first force of god.

In the process of stellar revolution, the change of orbital potential will be represented by elliptical orbit motion. Therefore, the origin of elliptical orbit motion of stars is the change of orbital potential after the star is slightly disturbed.

Summary: through the above research, we found that the star's rotation and revolution of origin from the center of gravity of the stars perturbation, leading to other stars around potential and the change of the rail potential, this kind of change, respectively, in the form of rotation and revolution of expression comes out, does not need god first driving force (the original kinetic energy that is to say, even if the stars, the stars of the original kinetic energy can only decide which orbit stars in potential plane rotation and revolution, and will not lead to the stars of rotation and revolution. Astral rotation 
and revolution do not need the original kinetic energy). At the same time, the origin of elliptical orbital motion of stars is that the orbital potential of stars changes in the form of elliptical orbital motion. Finally, we find that the rotation and revolution of stars do not have internal kinetic energy, nor do they need kinetic energy, but only a change of potential energy. Therefore, I explain the dynamic origin of the rotation and revolution of stars, as well as the origin of elliptical orbit motion of stars.

\section{3: origin of free fall motion}

Aristotle believed that heavy objects fall faster than light objects, and the greater the mass of an object, the faster it falls. In 1636 Galileo proposed the law of free fall motion. He believed that light objects and heavy objects landed at the same time. Although Galileo's law of free fall motion can describe the phenomena and laws of free fall motion, it cannot explain the origin of free fall motion. So, he knows that the motion of an object is independent of its mass, but he doesn't know why its falling speed is independent of its mass. What causes light objects and heavy objects to land at the same time?

According to the above derivation, we know that space is essentially a gravitational potential gradient field. The apple falls down because it is pushed by the potential energy from high altitude. So, the free fall motion of an object is actually the downward motion along the gradient space due to the thrust of the high potential energy.

At the same time, let's consider the question: are there any forces and counterforces between the two blackboard erasers in the falling process of free fall motion? When we studied physics in high school, we found that there is no force or reaction between two superimposed blackboards in free fall motion because they have the same acceleration. In other words, in the process of free fall movement, the two superimposed blackboard erasers in the hand cannot be regarded as a whole, and their mass cannot be superimposed.
We can enlarge the reasoning: because the eraser is atoms, even the same an eraser in the process of free fall, between the upper and the lower layer atoms on the blackboard eraser, actually there is no action and reaction, also a blackboard eraser in free fall in the process can be seen as a heap of independent of each other in the free fall of the atom, so the quality between atoms and atomic cannot achieve, therefore, an eraser free fall, its speed has nothing to do with the quality of an eraser.

Considering that the falling speed of all kinds of materials is the same, we can define the object of action of high potential energy as the same minimum unit of all kinds of materials: matrix $d v$. Since the mass of an object cannot be superimposed in the process of free fall motion, it can be concluded that the object of gravity is not the overall mass $M$, but the individual matrix of the component $d v$. Therefore, the object must be independent of the overall mass $M$ of the object in the process of free fall motion. This is the origin of free fall motion.

The mathematical expression is:

$$
g n d v \Rightarrow M g(M=n d v)
$$

Where, $d v$ refers to the matrix, $n$ is the number of substrates, $M$ is the total mass of the object, and $g$ is the gravitational acceleration, which is collectively expressed as: the motion of free falling body is that the substrates $d v$ of the substance are pushed down along the gradient space by the high potential energy, which is expressed in the form of overall falling at a macro level. Therefore, I explained the origin of the free fall movement from the micro level.

As can be seen from the above formula, we predict the existence of a new substance, namely, "ground substance".

\section{5: Conclusion}

This paper aims to establish a physical description of the general relativity and Newtonian 
gravity at the microscopic level (a new theory of quantum gravity) in order to seek a feasible way about the origin of gravity, while establishing a connection with dark matter and dark energy. Based on the physics principle of Newtonian mechanics and analytical mechanics, this paper establishes a new paradigm theory independent of Newtonian mechanics and analytical mechanics by introducing field theory model. It can solve some pain points in Newtonian mechanics and analytical mechanics. In the end, because this article interprets the origin of the movement, it can interpret the origin of inertia, ellipsoidal orbital motion, star rotation and revolutionary dynamics, and eliminate the confusion of God's first driving force.

In Newtonian mechanics, gravity is a kind of pull; in Einstein's general theory of relativity, gravity is not a basic force, but a manifestation of curved space-time; In this paper, gravity is further interpreted at the microscopic level as the spatiotemporal pressure generated by the gradient of space-time density. In other words, Newton's theory of gravity is an algebraic expression of a more basic theory of gravity; and general relativity is a geometric expression (interpretation) of a more basic theory of gravity.

In addition, the framework of my whole theory is to express the understanding of motion and gravity from the perspective of field theory. At the microscopic level, the gravitational field is further interpreted as the gradient field of space-time density, and gravity is further interpreted as the potential pressure (space-time repulsion or space-time pressure) generated by the gradient of density; the flection time-space is the embodiment of the gradient distribution of spatial density. Dark matter is the embodiment of space-time, and dark energy is the embodiment of the potential pressure (space-time repulsion) generated by the gradient of space-time density. The motion requires a potential difference to maintain, motion is the embodiment of the potential change of the object; inertia is the buffering process in which the kinetic energy of the object is consumed by the gravity of the gravitational field; the elliptical orbital motion is a macroscopic phenomenon manifested by the change of the orbital potential of the star after being subjected to force; the star's rotation and revolution of origin from the center of gravity of the stars perturbation, leading to other stars around potential and the change of the rail potential, this kind of change, respectively, in the form of rotation and revolution of expression comes out; the motion of free falling body is that the substrates of the substance are pushed down along the gradient space by the high potential energy(potential pressure), which is expressed in the form of overall falling at a macro level, Therefore, the free fall motion has nothing to do with the quality of the object.

Finally, frankly speaking, there is also a pain point in this article, that is, the lack of corresponding calculations to support my point of view. This is because I am not good at computing research; on the other hand, because I don't know how to solve this pain point now. Therefore, I hope that interested friends can help me solve this problem, and I hope to get the reviewer's understanding.

\section{Reference}

[1] A. M. Baranov. "Gravitational fields of lightons and helixons in General Relativity." Gravitation \& Cosmology 12(2011).

[2] Aharony, O. et al. "Large N Field Theories, String Theory and Gravity." Physics Reports 323.3-4(1999):183-386.

[3] T. Damour, and A. M. Polyakov. "String theory and gravity." General Relativity \& Gravitation (1994).

[4] Jaume Giné. "On the Origin of the Inertial Force and Gravitation." International Journal of Theoretical Physics50.2(2011):607-617.

[5] Schaff, Jacob. "Gravitation: What it really 
is." Physics Essays. 22.4(2009).

[6] Schaff, Jacob. "Spacedynamics: The Origin of the Inertial Dynamics Within Gravitational Fields." Physics Essays 16.2(2003):-

[7] B. Mashhoon "On the origin of inertial accelerations."

Nuovo

Cimento

B 109.2(1994):187-199.

[8] Nicholas, Ionescu-Pallas. "Reflections concerning the genuine origin of gravitation." Romanian Reports in

Physics 55(2003):7-48.

[9] Graneau, P, and Graneau, N. "Machian Inertia and the Isotropic Universe." General Relativity \& Gravitation 35.5(2003):751-770.

[10] Sonego, Sebastiano, and M. Massar. "On the notions of gravitational and centrifugal force in static spherically symmetric space-times." Monthly Notices of the Royal Astronomical Society 281.2(1996):659-665.

[11] Winterberg, and Friedwardt. "The Gravitational Origin of the Higgs Boson Mass." Zeitschrift für Naturforschung A 69.5-6(2014).

[12] Masahiro, Ibe, and T. T. Yanagida. "The lightest Higgs boson mass in pure gravity mediation model." Physics Letters B 709.4-5(2012):374-380.

[13] Ascasibar Y, Yepes G, S. Gottlöber, et al. On the physical origin of dark matter density profiles[J]. Monthly Notices of the Royal Astronomical Society, 2010, 352(4):1109-1120.

[14] Dekel A, Silk J. The origin of dwarf galaxies, cold dark matter, and biased galaxy formation[J]. Astrophysical Journal, 1986, 303(1):39-55.

[15] Nojiri, S., \& Odintsov, S. D. (2007).
Introduction to modified gravity and gravitational alternative for dark energy. International Journal of Geometric Methods in Modern Physics, 04(01), 115-145.

[16] Tsujikawa S. Matter density perturbations and effective gravitational constant in modified gravity models of dark energy[J]. Physical Review D, 2007, 76(2):-

[17] Li M. A Model of Holographic Dark Energy[J]. Physics Letters B, 2004.

[18] Amendola L, Gannouji R, Polarski D, et al. Conditions for the cosmological viability of $f(R)$ dark energy models[J]. Phys.rev.d, 2006, 75(8):307-309.

[19] Tsujikawa S. Modified gravity models of dark energy[J]. 2010.

[20] Hsu S D H. Entropy Bounds and Dark Energy[J]. Physics Letters B, 2004.

[21] Erik, Verlinde. "On the origin of gravity and the laws of Newton." Journal of High Energy Physics (2011).

[22] R. M. Wald, "General Relativity," The University of Chicago Press, 1984

[23] San-Hui Zhang, College physics (Tsinghua university press, Beijing 9,2008 ) 